\begin{tabular}{c|c|c}
\hline \hline & CLIMATE RESEARCH & Published August 8 \\
Vol. 22: 1-11, 2002 & Clim Res & \\
\hline
\end{tabular}

\title{
Recent and future modulation of the annual cycle
}

\author{
Craig J. Wallace*, Timothy J. Osborn
}

Climatic Research Unit, School of Environmental Sciences, University of East Anglia, Norwich NR4 7TJ, United Kingdom

\begin{abstract}
This study investigates changes to the annual temperature cycle in both observed records and output from a coupled ocean-atmosphere global climate model. Using least-squares harmonic analysis, changes to the observed annual harmonic (for the time period 1856-1998), in addition to the 1961-1990 climatology, are compared with 9 simulations from the HadCM2 model. The first simulation is a $1400 \mathrm{yr}$ control integration, whilst the remainder are from 2 ensembles representing (1) increases in $\mathrm{CO}_{2}$ concentrations and (2) a combination of $\mathrm{CO}_{2}$ and sulphate aerosol increases. Observed and simulated climatologies are generally comparable, although large amplitude and phase discrepancies exist over northern North America and high-latitude oceans, respectively. The agreement may be partly artificial over the oceans due to the use of flux adjustments to maintain a realistic sea-surface temperature field. Observed northern hemisphere amplitude decreases during the 20th century agree well with simulated changes, although there are some regional differences; observed changes to the southern hemisphere amplitude are insignificant. The sign of northern hemisphere phase changes are opposite in the 2 data sets. The nature of these results is unchanged after consideration is given to the varying spatial coverage of the observed data set, by means of applying a frozen grid mask to both observed and simulated data. These findings are consistent with previous studies, though we extend them by updating the observed record, by using ensembles to better define the climate change signal, and by considering the direct effects of sulphate aerosols. For a given warming, the inclusion of aerosols results in an enhanced amplitude decrease within the northern hemisphere, related to the summertime maximum of the direct sulphate cooling effect.
\end{abstract}

KEY WORDS: Annual temperature cycle $\cdot$ Greenhouse gas $\cdot$ Sulphate aerosols $\cdot$ Climate change Phase - Amplitude

Resale or republication not permitted without written consent of the publisher

\section{INTRODUCTION}

Changes to the annual cycle of temperature are important from both climatic and biological points of view. Climatically, the cycle is responsible (directly or indirectly) for controlling a number of other variables, such as pressure patterns and circulation characteristics. Annual variations of temperature are greater than most interannual variations, yet the annual cycle is frequently removed (by considering anomalies from it) in studies concerning climate variability or change. From a biological perspective, the annual cycle is paramount in dictating the timing of natural events (phenology),

*E-mail: craig.wallace@uea.ac.uk some of which are critical to animals, whilst others are important for agriculture.

To date, only a small number of studies have served to redress this neglect of the annual cycle. Thomson (1995) showed how observed 20th century changes to the phase of the annual cycle cannot be exclusively explained by insolation variability and argued for an anthropogenic greenhouse signal in the northern hemisphere $(\mathrm{NH})$. Other work has analysed extended European temperature records, revealing that the most significant changes in the annual cycle occurred during the 1920s, with a distinct decrease in annual cycle amplitude (Thompson 1995).

Mann \& Park (1996) investigated changes to the NH observed annual harmonic for the period 1854-1990 
and compared the results with changes diagnosed from 2 general circulation climate models, i.e. the Geophysical Fluid Dynamics Laboratory (GFDL) coupled ocean-atmosphere model and the National Center for Atmospheric Research (NCAR) Community Climate Model (CCM1) general circulation model, coupled with a non-dynamic, mixed-layer ocean. A 100 yr simulation of the GFDL model was used, forced with a gradual $\left(1 \% \mathrm{yr}^{-1}\right)$ increase in $\mathrm{CO}_{2}$ concentrations, whilst the equilibration change in the annual cycle of the CCM1 model, between 330 and $460 \mathrm{ppm} \mathrm{CO}_{2}$ was examined. The response of both models under enhanced $\mathrm{CO}_{2}$ forcing exhibits a decrease in amplitude accompanied by phase delays, with the magnitude of both changes stronger at higher latitudes. Mann \& Park (1996) attributed these trends to a strong icealbedo feedback. Observed changes in amplitude are consistent with model simulations, yet phase trends are of opposite sign (i.e. observed phase advances, but a simulated delay). On the one hand, this discrepancy may be related to the lack of high-latitude sampling in the observed data used, with observed phase advances being the manifestation of some non-greenhouserelated variability; a spatially complete data set might therefore produce an average $\mathrm{NH}$ response that agrees with the model simulations. On the other hand, Mann \& Park (1996) flagged the possibility that observed phase advances in mid-latitudes are indeed a signal of enhanced greenhouse gases, and thus flaws in the models, especially within these regions, might exist.

In a recent study, Stott et al. (2001) tried to detect, in the observed temperature record, the signals of externally forced climate change, simulated by the Hadley Centre's HadCM2 climate model (Johns et al. 1997). They found that, for some signals, such as solar forcing, detection was more convincing if information about seasonality changes was utilised in addition to the annual-mean temperature change. This implies some consistency between observations and the model simulations, though Stott et al. (2001) only consider large spatial scales and do not present patterns or time series of seasonality change, so we are unable to interpret them in terms of physical mechanisms.

This paper builds on and extends the work of Mann \& Park (1996). We consider the spatially complete, globally gridded climatology (1961-1990) of Jones et al. (1999). We also diagnose temporal changes for both hemispheres, using data updated to 1998. Observed changes in amplitude and phase are compared with simulations from the HadCM2 global climate model, under enhanced anthropogenic forcing. Not only do we examine the response of the annual harmonic to rising greenhouse gas concentrations, but also to a combination of greenhouse gas and sulphate aerosol variation. Furthermore, we make use of ensembles of simulations to provide a more robust identification of the climate change signal.

\section{DATA AND METHODS}

\subsection{Model output data}

We use monthly $1.5 \mathrm{~m}$ air temperature from 9 simulations of the HadCM2 coupled climate model (Johns et al. 1997). This is a coupled ocean-atmosphere model, incorporating a thermodynamic sea-ice model. Model data is resolved on a $2.5^{\circ}$ latitude by $3.75^{\circ}$ longitude global grid. Of particular importance to this study is that heat and freshwater flux adjustments are applied during the coupling of the atmosphere and ocean components. Though these adjustment terms are constant in time, they do vary with the month of the year, having being diagnosed to maintain a more realistic mean climate and mean annual cycle under constant external forcing than would otherwise have been obtained. We might expect, therefore, close (but partly artificial) agreement between model and observations in terms of the mean annual cycle over the oceans.

A $1400 \mathrm{yr}$ control simulation with fixed forcing (based upon present-day levels of greenhouse gases) is used to assess the statistical significance of externally forced changes (Tett et al. 1997). The remaining simulations come from 2 ensembles (G and GS), each of 4 members, all covering a 239 yr time slice, running from 1861 through 2099 inclusive (Mitchell et al. 1999). The G ensemble simulations are forced from 1861 to 1990 with historical increases in $\mathrm{CO}_{2}$ concentrations and thereafter with a $1 \% \mathrm{yr}^{-1}$ increase. Forcing in the GS ensemble is similar, but also includes the direct radiative effects of sulphate aerosols.

\subsection{Observed data sets}

Two observed data sets are used in this analysis. Both contain monthly-mean temperature observations on a $5^{\circ}$ by $5^{\circ}$ grid box basis. The first is a spatially complete 1961-1990 average climatology of land and marine $1.5 \mathrm{~m}$ air temperatures (Jones et al. 1999). In constructing these averages, some elevationdependent interpolation between grid boxes where observations are present is used to infill boxes where data are absent. The second data set comprises gridded, observed time series running from 1856 through 1998, expressed as anomalies from the 1961 to 1990 climatology. This is a blended data set of sea-surface temperature anomalies over oceans and $1.5 \mathrm{~m}$ air temperature anomalies over land (Jones et al. 1999). The spatial coverage of this second data set is incom- 
plete and varies temporally, i.e. interpolation is not used to infill missing grid boxes.

The spatially complete climatology of absolute temperatures can tell us about the present-day characteristics of the annual cycle (Section 3.1). To identify variations in the annual cycle through time, the climatology must be combined with the anomaly time series. This is done on a grid box by grid box basis, to obtain gridded time series of absolute temperature. This data set has incomplete spatial coverage that varies through time, but is adequate for identifying the spatial pattern of annual cycle changes (Section 3.3) for part of the globe at least.

The combined data set is not suitable for analysing changes in the annual cycle of hemispheric-mean temperature, however, because the results would be dominated by spurious trends associated with the changing spatial coverage of the data. This is a consequence of the fact that the spatial variability in the annual cycle parameters is much greater than their temporal variability. Instead, we compute hemispheric means of the climatology and of the temperature anomalies separately and then combine them to obtain hemispheric time series of absolute temperature. This approach prevents the changing coverage of the anomaly data set from introducing temporal changes in the baseline climatology, which could be quite large. Nevertheless, changing spatial coverage may still introduce small biases into the results, and this problem is dealt with in Section 3.2 by using a frozen grid (FG) of temperature anomalies.

\subsection{Methods}

The amplitude and phase of the annual harmonic are diagnosed using a least-squares regression transform, described by Wilks (1995). This method is based on a fundamental equation, describing temperature at time $t$ in a series $y$ as the result of the following harmonic function:

$$
y_{t}=\bar{y}+C \cos \left(\frac{2 \pi t}{n}-\phi\right)
$$

where $n$ is the period, $C$ the amplitude and $\phi$ the phase of that particular harmonic. Given that this harmonic can be expressed as the sum of an unshifted (i.e. zero phase) cosine and an unshifted sine wave, with amplitudes $A$ and $B$, respectively:

$$
C \cos \left(\frac{2 \pi t}{n}-\phi\right)=A \cos \left(\frac{2 \pi t}{n}\right)+B \sin \left(\frac{2 \pi t}{n}\right)
$$

we can transform Eq. (1) into:

$$
y_{t}=\bar{y}+A x_{1}+B x_{2}
$$

where $x_{1}=\cos (2 \partial t / n)$ and $x_{2}=\sin (2 \partial t / n)$. Given a set of values of $y$ at times $t$, the values of $x_{1}$ and $x_{2}$ can be evaluated and Eq. (3) can be solved by a multiple linear regression to obtain values of $\bar{y}, A$ and $B$ that give the best fit of the harmonic. Recovery of the amplitude of the annual harmonic is achieved by using:

$$
C=\left(A^{2}+B^{2}\right)^{1 / 2}
$$

and the phase is given by:

$$
\phi= \begin{cases}\tan ^{-1} \frac{B}{A} & A>0 \\ \tan ^{-1} \frac{B}{A} \pm \pi & A<0 \\ \frac{\pi}{2} & A=0\end{cases}
$$

For each analysis, 12 temperature values are provided for the regression procedure (1 for each month) at times specified as the centre of each calendrical month (i.e. $t_{1}=15.5, t_{2}=45.125$, days since January 1 ), taking account of leap years by assuming an annual cycle period of $n=365.25 \mathrm{~d}$ and $28.25 \mathrm{~d}$ for February. For the baseline climate and the spatial change patterns of the annual harmonic (Sections 3.1 and 3.3), these monthly values are $30 \mathrm{yr}$ means. For the analysis of hemispheric and global temperatures (Section 3.2), we are dealing with time series and, to damp some of the interannual variability, both observed and simulated time series are passed through a triangularly weighted $11 \mathrm{yr}$ moving window. We then apply the above method to obtain phase and amplitude values for the first harmonic of the annual cycle.

In order to elucidate any artificial trends in amplitude and phase caused by changing geographical coverage of observations, we construct a FG mask which is applied to both the observed and simulated time series data, in Section 3.2. Grid boxes included in the mask satisfy the following criteria: Firstly, each month of the year must be represented in more than $50 \%$ of the years in each observed decade (e.g. there must be more than 5 October values per decade). Secondly, each year of the series must contain at least 6 monthly observations to ensure that the months present are not clustered towards either the start or end of the decade. A map of those grid boxes which meet the above conditions, for the period 1900-1998 is shown in Fig. 1. 1900 is chosen as a starting year to allow for a suitable time series length (99 yr), whilst at the same time providing space for a reasonable observation network to have developed.

Trends in the diagnosed amplitude and phase time series are evident, and the null hypothesis that each trend could have occurred by chance is tested by 2 significance tests (against the alternative hypothesis that the trend is unusual, given the variability evident in 


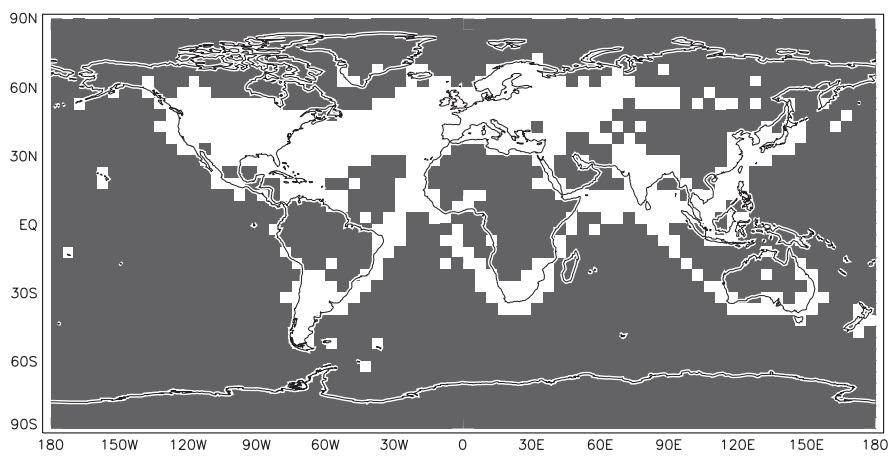

Fig. 1. Map of grid boxes (white) included in the frozen grid mask

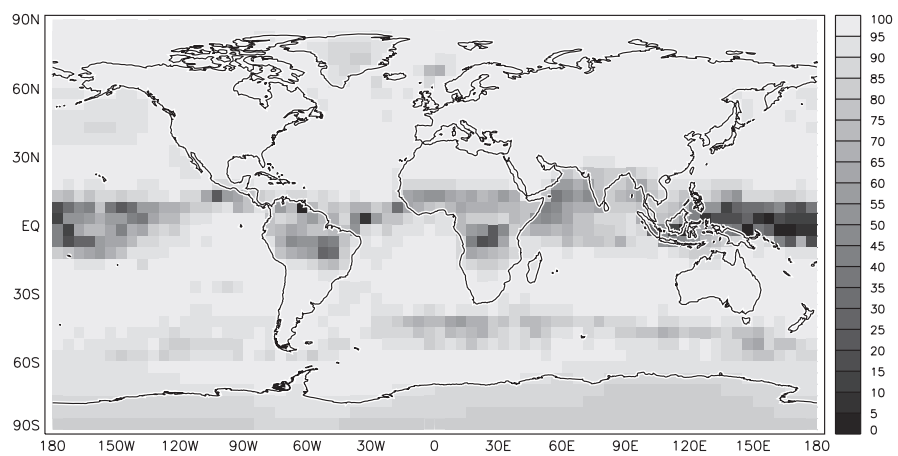

Fig. 2. Percent variance accounted for in the annual temperature cycle by the first harmonic

the time series, for Test I, or the variability evident in the HadCM2 control run, for Test II). The first of these (Test I) is a conventional $t$-test of the correlation coefficient between the values and time. We account for any autocorrelation present in the time series by reducing the effective independent sample size with a method described by Wilks (1995; Eq. 5.12). As the simulated amplitude and phase time series are derived from an ensemble mean, and thus likely to have an artificially high autocorrelation, the lag-1 correlation coefficients from the observed time series are applied when calculating the corrected simulated sample size. Test II compares trends with those extracted from the control run of HadCM2; many (overlapping) segments of simulated temperatures, of the same length as the trend to be tested, are each analysed in the same way to build up a distribution of trends in amplitude or phase that might be possible with no change in external forcing. Test II is not constrained by the assumptions of normality and the simple adjustment for serial correlation required by Test $I$, but it is necessary to assume that the $1400 \mathrm{yr}$ model control run provides a realistic gauge of annual cycle variability.

With reference to the spatially complete 1961-1990 observed climate baseline, the annual harmonic is found to be sufficient in accounting for over $90 \%$ of the variance in the annual cycle of temperature for most of the planet (Fig. 2). Exceptional locations include the tropics and parts of the southern hemisphere $(\mathrm{SH})$ where the annual cycle assumes a non-sinusoidal form, e.g. over Antarctica, where the period of time during each winter with constant (zero) insolation and the existence of a semi-annual variation in circulation (van den Broeke 1998), both act to cause a deviation of temperature from the annual harmonic. These, and other regions are discussed further by May et al. (1992).

\section{RESULTS}

\subsection{Baseline climatology (1961-1990)}

Table 1 displays observed and simulated seasonal cycle characteristics for the 1961-1990 reference period. This comparison is made using the spatially complete observed data set and the mean of the ensemble mean temperatures for the corresponding simulated time period (i.e. 1961-1990). These values are the amplitudes and phases of hemispheric-mean temperatures rather than the hemispheric-mean of the grid-box amplitudes and phases. There are some discrepancies between amplitude values for the northern and, to a lesser degree, southern hemispheres. Consultation with globally gridded differences (Fig. 3a) reveals that NH amplitudes (especially in the proximity of the Hudson Bay area, Canada) are underestimated by as much as $15^{\circ} \mathrm{C}$, contributing to a hemispheric average $0.64^{\circ} \mathrm{C}$ below the observed amplitude. This is a manifestation of the tendency of the model to underestimate minimum winter temperatures in high-latitude areas. Conversely, simulated amplitudes in the SH are overestimated especially around continental Antarctica, though the amplitude of the hemispheric mean is almost identical to that of the observed. The reason for the apparent Antarctic errors requires further investigation, though it should be noted that this is a region of scarce observational data, and hence many observed values are interpolated.

Table 1. Observed and simulated amplitude $\left({ }^{\circ} \mathrm{C}\right)$ and phase (date of peak temperature after January 1) for the baseline 1961-1990 period in the northern (NH) and southern (SH) hemispheres. Simulated values are the average value for each ensemble averaged

\begin{tabular}{|lrr|}
\hline & $\mathrm{NH}$ & \multicolumn{1}{c|}{$\mathrm{SH}$} \\
\hline Observed amplitude & 6.68 & 2.85 \\
Simulated amplitude & 6.04 & 2.88 \\
Observed phase & 205.66 & 30.42 \\
Simulated phase & 206.95 & 31.97 \\
\hline
\end{tabular}


a

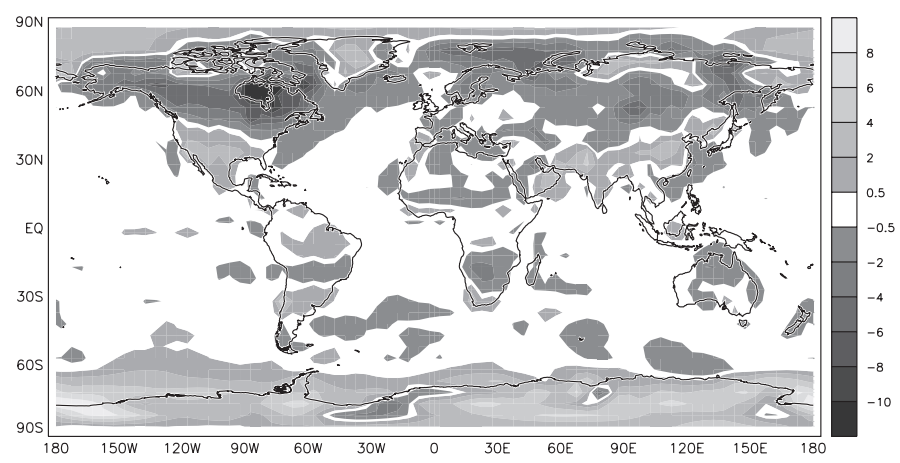

b

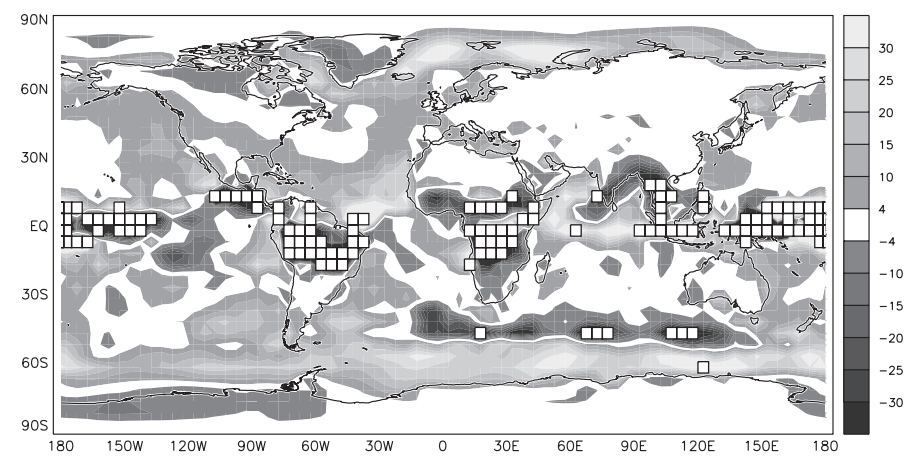

Fig. 3. Differences between simulated and observed 1961-1990 (a) amplitude $\left({ }^{\circ} \mathrm{C}\right.$ ) and (b) phase (days). In (b), white boxes indicate a phase difference of more than $40 \mathrm{~d}$, though in the tropics this is not important because the annual harmonic explains only a small part of the annual cycle (see Fig. 2)

Model annual cycle phases (days after January 1) for both hemispheres show delays over observed phases: 1.29 and $1.55 \mathrm{~d}$ for the $\mathrm{NH}$ and $\mathrm{SH}$, respectively. Fig. 3b shows vicinities with considerable phase delays (i.e. positive differences) in the model simulations, particularly in the Southern Ocean. We may speculate (on the basis of the location of the main discrepancies) that these phase delays are related to the incorrect simulation of sea-ice cover. Regions of disagreement are also evident for a more limited area in the northern North Atlantic and Barents Sea, again near to the region with seasonal sea-ice cover. Phase differences in tropical regions are relatively unimportant and should be ignored (those errors greater than $40 \mathrm{~d}$ in magnitude are left white in Fig. 3b). Exclusion is a consequence of the double-peak nature of tropical annual cycles, resulting from the semi-annual transit of the overhead sun. The phase value of the fundamental harmonic is dependent on which peak is dominant, and for many grid boxes, it is not the same in both simulated and observed cycles. The results of such minor discrepancies can be phase differences on the order of $100 \mathrm{~d}$ or more.

It is quite noticeable (Fig. 3b) that the phase of the simulated annual harmonic is more realistic over extratropical land than extra-tropical oceans. This is likely to be because of the relative ease with which the land heat capacity is modelled, compared with the more dynamic heat capacity of the oceanic mixed layer. It is perhaps surprising that the artificial flux adjustments that are applied in HadCM2 over the oceans do not force the annual cycle to be more realistic, given that they successfully force a realistic annual cycle amplitude (Fig. 3a). Even away from the sea-ice affected regions, significant phase errors occur. Pierce (1996) explains how phase delays can be introduced when an ocean model is forced using a relaxation to climatology as a surface boundary condition; such a condition is used in deriving the flux adjustments in HadCM2, so this could explain the phase errors over the oceans.

\subsection{Hemispheric time series}

In addition to an evaluation of the model's simulation of present-day climate, it is also of interest to diagnose the response of the annual harmonic to changing external forcing. In the first instance, we analyse the annual harmonic of hemispheric-mean temperature from the ensemble-mean of the G and GS simulations, and from the observations, using all available data, i.e. complete data from the simulations and a time-dependent coverage of observed data, denoted the unfrozen grid (UFG). Due to the systematic differences between simulated and observed annual cycles, observed and simulated results are expressed as deviations from their $50 \mathrm{yr}$ reference mean (1866-1915). Thus, we concentrate on changes through time in the various data sets.

In both simulated and observed time series, the $\mathrm{NH}$ shows a decline in amplitude (Fig. 4a). Since the 1950s, all observed amplitudes have been beneath the 1866-1915 mean. The linear trend in the final $60 \mathrm{yr}$ of the observed time series (1934-1993, where 1993 is the value from the $11 \mathrm{yr}$ window spanning 1988-1998) is $-0.12^{\circ} \mathrm{C}$ per $60 \mathrm{yr}$, significant at the $95 \%$ level in both Tests I and II (Table 2). This is remarkably close to the trends over the same time period in the G and GS ensemble means (Table 2), which are also significant in both Tests I and II. Phase values demonstrate less agreement (Fig. 4b): whilst observed data indicates an advance of $0.76 \mathrm{~d}$ (significant only in Test I) over the last $60 \mathrm{yr}$ (though an opposite trend prior to this), both model ensembles exhibit phase delay trends of $0.84(G$, significant in Tests I and II) and 0.33 (GS, significant in Test I) d per 60 yr. G forced model projections also predict an earlier onset of noticeable phase delays. Projections for the end of the model simulations suggest a decrease in amplitude in the region of $0.45^{\circ} \mathrm{C}\left(0.46^{\circ} \mathrm{C}\right.$ in $\mathrm{G}$ and $0.45^{\circ} \mathrm{C}$ in GS) and phase delays approaching $3 \mathrm{~d}$ (2.96 d in G and $2.69 \mathrm{~d}$ in GS), for the mean $\mathrm{NH}$ temperature. 

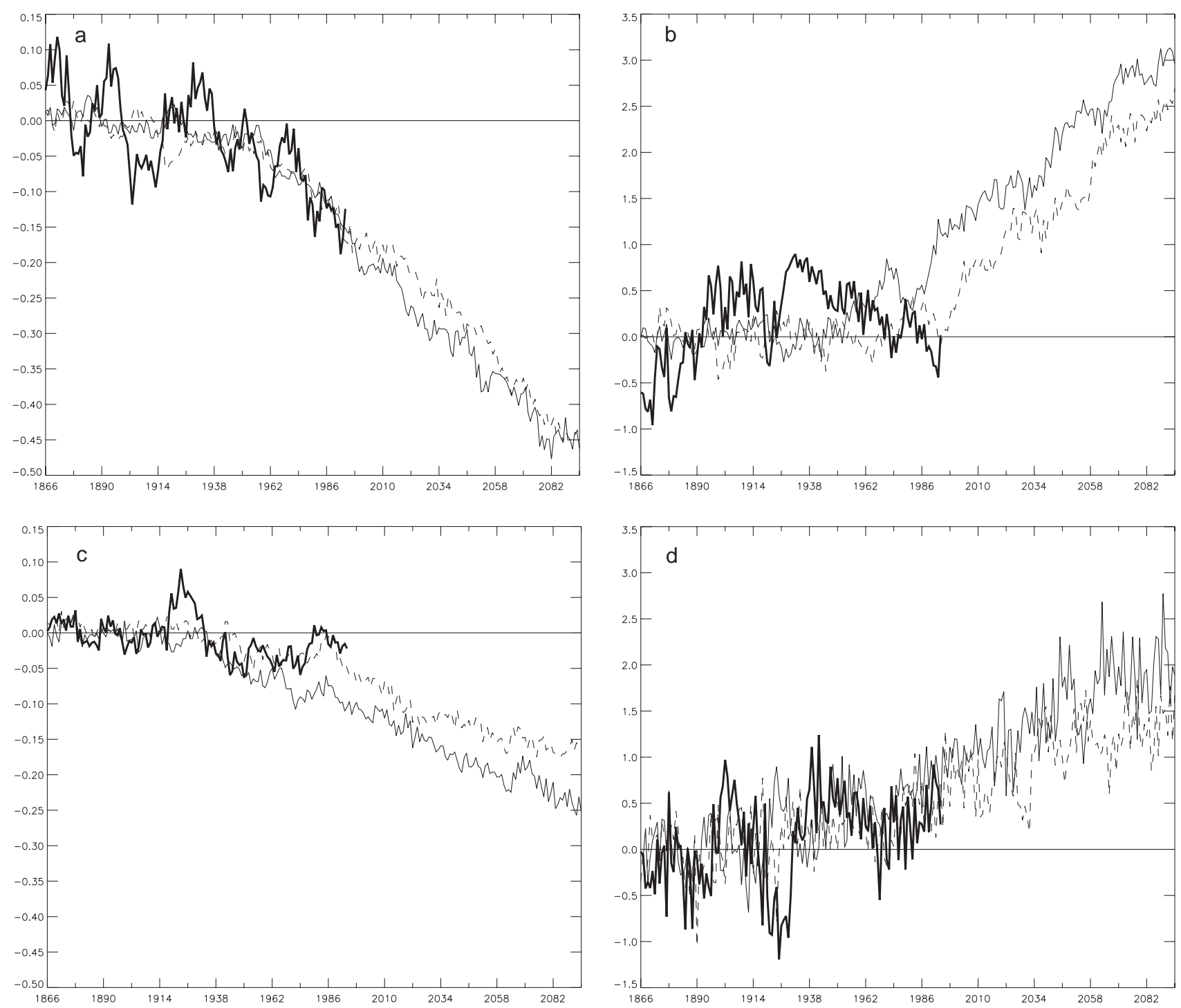

Fig. 4. Changes in $(\mathrm{a}, \mathrm{c})$ amplitude $\left({ }^{\circ} \mathrm{C}\right)$ and $(\mathrm{b}, \mathrm{d})$ phase (days) of the annual cycle of $(\mathrm{a}, \mathrm{b}) \mathrm{NH}$ and $(\mathrm{c}, \mathrm{d})$ SH temperature: observed (thick lines) and means from the G (thin lines) and GS (dashed lines) ensembles

Observed $\mathrm{SH}$ amplitude changes are insignificant (Fig. 4c), whilst simulated changes, both indicating decreases for the last $60 \mathrm{yr}$, are significant in Test I for both G and GS simulations, and in Test II for G only. The amplitude decreases become larger and much more significant as the simulations progress into the future. There is a sizeable difference in the G and GS amplitude responses by the end of the simulations, with the former exhibiting a $0.25^{\circ} \mathrm{C}$ decrease compared to $0.16^{\circ} \mathrm{C}$ in the latter, by 2094 .

The difference between the G and GS amplitude changes is much larger in the $\mathrm{SH}$ than in the $\mathrm{NH}$, which is initially surprising given that the sulphate aerosol forcing (the only difference between the ensembles) is concentrated in the NH. To investigate this further, we plot in Fig. 5 the hemispheric amplitude changes against the hemispheric-mean temperature change of each ensemble. Thus we can compare how the amplitudes change with climate state, rather than with time. For the SH (Fig. 5b), the relationship between amplitude and mean temperature is similar in both ensembles, demonstrating that the different amplitude changes (Fig. 4c) are simply due to different warming rates in $\mathrm{G}$ and GS. For the $\mathrm{NH}$, the GS ensemble shows a greater decrease in amplitude per degree of warming than does the G ensemble (Fig. 5a), and therefore the similar rates of amplitude decrease (Fig. 4a) in the 2 ensembles are because the G ensemble warms more rapidly. The reason for the enhanced amplitude decrease in the GS simulation is most likely 
Table 2. Linear trends $\left({ }^{\circ} \mathrm{C}\right.$ per $60 \mathrm{yr}$ for amplitude, days per $60 \mathrm{yr}$ for phase) for observed and simulated annual cycle time series (1934-1993). G and GS: greenhouse and greenhouse plus sulphate forcings, respectively

\begin{tabular}{|c|c|c|c|c|c|}
\hline & & & nplitude & & Phase \\
\hline & & Trend & Significance test (95\%) & Trend & Significance test (95\%) \\
\hline $\mathrm{NH}$ & Observed & -0.12 & I \& II & -0.76 & I \\
\hline & G & -0.13 & I \& II & 0.84 & I \& II \\
\hline & GS & -0.12 & I \& II & 0.33 & I \\
\hline $\mathrm{SH}$ & Observed & 0.02 & - & 0.03 & - \\
\hline & G & -0.07 & I \& II & 0.69 & I \\
\hline & GS & -0.02 & I & 0.26 & I \\
\hline
\end{tabular}
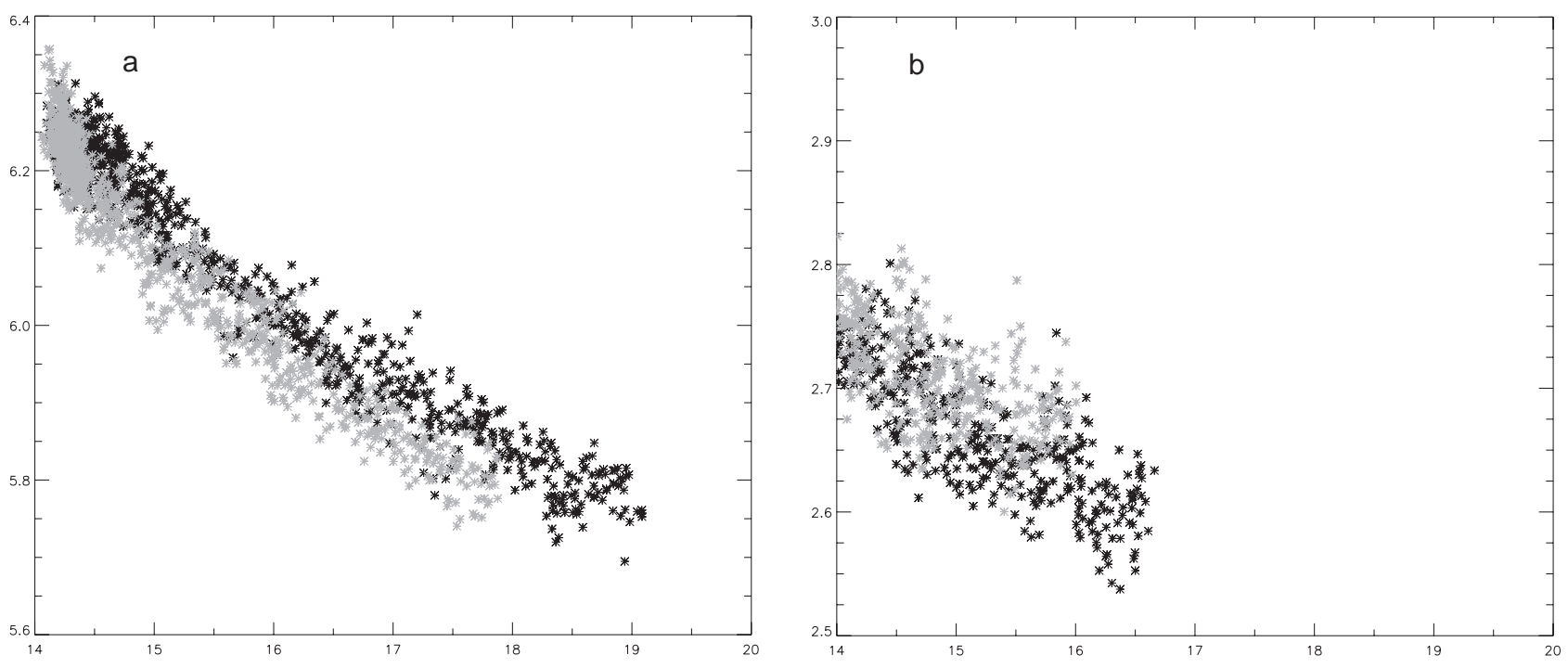

Fig. 5. Mean-hemispheric amplitude $\left(y\right.$-axis, $\left.{ }^{\circ} \mathrm{C}\right)$ plotted as a function of mean-hemispheric temperature $\left(x\right.$-axis, $\left.{ }^{\circ} \mathrm{C}\right)$ for $(\mathrm{a}) \mathrm{NH}$ and (b) SH, for both $\mathrm{G}(*)$ and GS (*) simulations

due to the direct radiative influence of the sulphate aerosols (in HadCM2 this is parameterised by an increase in surface albedo) having its greatest cooling effect during NH summer. Thus, winters will warm relative to summers, and the annual harmonic amplitude will decrease. This is in addition to the greater winter warming (relative to summer) that already occurs under greenhouse gas forcing. This latter warming is in part due to the ice and snow albedo feedback that is particularly important during the cold half of the year. As ice and snow cover are reduced, it is anticipated that this positive winter feedback will reduce in strength and the differential rate of winter and summer warming will decrease. This is evident in the curvature of the relationship between amplitude change and temperature change in Fig. 5a.

Phase delays are evident (Fig. 4d) in all $3 \mathrm{SH}$ time series (significant in Test I, for the simulated series, but only because the ensembles strengthen the signal-tonoise ratio), although results show greater interannual variability than for the $\mathrm{NH}$. The observed delay $(0.03$ d), however, falls short of both the G (0.69 d) and GS (0.26 d) simulated trends (Table 2), though observational coverage is poor and varies through time. Phase delays increase towards the end of the simulations, with final delays of around $1.7 \mathrm{~d}(1.89 \mathrm{~d} \mathrm{G}$ and $1.66 \mathrm{~d}$ GS), compared with the 1866-1915 mean values.

These hemispheric results are based on a full coverage of model output and incomplete time-varying observational coverage. We have not, therefore, performed a like-with-like comparison of simulated and observed changes, and it is also possible for artificial trends to occur in the observed time series, though the use of only anomalies from the time-varying coverage reduces the likelihood of large artificial trends. The FG analysis (see Section 2.3) allows a like-with-like comparison and prevents artificial trends, by applying the same fixed mask to all data sets. The FG (Fig. 1) includes only $21 \%$ of all grid boxes. Hemispheric means calculated from just these grid boxes will have higher 
Table 3. Linear trends $\left({ }^{\circ} \mathrm{C}\right.$ per $60 \mathrm{yr}$ for amplitude, days per $60 \mathrm{yr}$ for phase) for observed and simulated annual cycle time series (1934-1993) using the frozen grid

\begin{tabular}{|c|c|c|c|c|c|}
\hline & & \multicolumn{2}{|c|}{ Amplitude } & \multicolumn{2}{|r|}{ Phase } \\
\hline & & Trend & Significance test $(95 \%)$ & Trend & Significance test (95\%) \\
\hline \multirow[t]{3}{*}{$\mathrm{NH}$} & Observed & -0.21 & I \& II & -1.40 & I \& II \\
\hline & G & -0.06 & I & 0.85 & I \& II \\
\hline & GS & -0.12 & I \& II & 0.51 & I \\
\hline \multirow[t]{3}{*}{$\mathrm{SH}$} & Observed & 0.01 & - & 0.91 & I \\
\hline & G & 0.02 & I & 0.39 & I \\
\hline & GS & 0.05 & I & 0.06 & - \\
\hline
\end{tabular}

variability, and thus trends might be less significant. This is especially true for the $\mathrm{SH}$, where only $9 \%$ of grid boxes are present in the FG.

Analogous 60 yr trend results for observed and simulated FG data are shown in Table 3. Meanwhile, Fig. 6 displays time series data as deviations from the first 50 yr mean (in this case, 1905-1954). FG-observed $\mathrm{NH}$ results exhibit a greater decrease in amplitude than corresponding UFG results (compare $-0.21^{\circ} \mathrm{C}$ by 1993 with $-0.12^{\circ} \mathrm{C}$ ), in contrast with model FG amplitude trends, which suggest a smaller decrease (the FG removes much of the Arctic region, where the majority of the simulated amplitude decrease occurs). Similarly, a greater phase advance is seen in the observed FG results, whereas simulated data continues to suggest delays closer to the simulated UFG results. Little change is found in the SH FG (comprising only a small number of grid boxes) over the historical period, although sizeable amplitude increases can be seen in model projections by $2094\left(0.21^{\circ} \mathrm{C}\right.$ in $\mathrm{G}$ and $0.18^{\circ} \mathrm{C}$ in GS), which are of opposite sign to those found for the UFG (i.e. complete hemispheric coverage). Clearly the observational coverage for the full 20th century (i.e. the FG) is too small to measure the expected SH amplitude change signal. Significant (Test I) delays are evident in observed and $\mathrm{G}$ phase results.

\subsection{Global-gridded results}

Spatial patterns of change to the annual cycle are presented in Fig. 7. Here, average annual cycle values for 1909-1938 have been subtracted from average 1969-1998 values. Observed grid boxes are excluded from the calculation if each month is not represented in at least 21 of the 30 years in each time window. These 2 specific time windows are selected to represent average conditions at both the start and close of the last century. Selecting a window length of $30 \mathrm{yr}$ ensures that highfrequency changes do not mask the overall trend, as they might, for instance, if a 5 or 10 yr window were selected.
Sizeable NH continental amplitude decreases are visible in Fig. 7a. The most extreme of these are located in central and eastern regions of Russia and approach $-3^{\circ} \mathrm{C}$ in size. A region of smaller change is found in western Russia. Elsewhere, there are few grid boxes which exceed $\mathrm{a} \pm 0.5^{\circ} \mathrm{C}$ change. Changes in both the $G$ and GS simulations suggest widespread amplitude decreases in the northern high latitudes, with additional decreases around the Antarctic coast, both of which are regions with poor observational coverage. The magnitude of these changes is greater in the $G$ simulation, but does not exceed $-3^{\circ} \mathrm{C}$. GS results also indicate decreases across much of the globe, up to $0.5^{\circ} \mathrm{C}$, especially within the region of central Canada and parts of Europe. Some amplitude decreases are apparent in central and eastern regions of Russia, although in neither ensemble does the magnitude of change match the observations. Using those grid boxes with observational data present as a mask (though the tropics, defined as the area between $25^{\circ} \mathrm{N}$ and $25^{\circ} \mathrm{S}$, are removed in all cases), pattern correlations between the observed, G and GS changes were calculated, providing more information on the agreement of the patterns of change. Observed changes show a 0.24 correlation with the $G$ simulation changes and a 0.11 correlation with GS changes. When compared with each other, G and GS changes have a correlation coefficient of 0.53 . However, due to the spatial autocorrelation which exists between the grid boxes, and the problems associated with quantifying this, the effective degrees of freedom remain unknown. This prevents the significance of these correlations being tested by traditional methods.

We can compare the magnitude of changes by calculating the area-mean (again with tropical areas excluded) root mean square (rms) deviation. The observed rms deviation is 0.37 , indicating that the magnitude of observed amplitude change is greater than those in the model simulations for the same period (compare with 0.25 and 0.22 for G and GS respectively). 

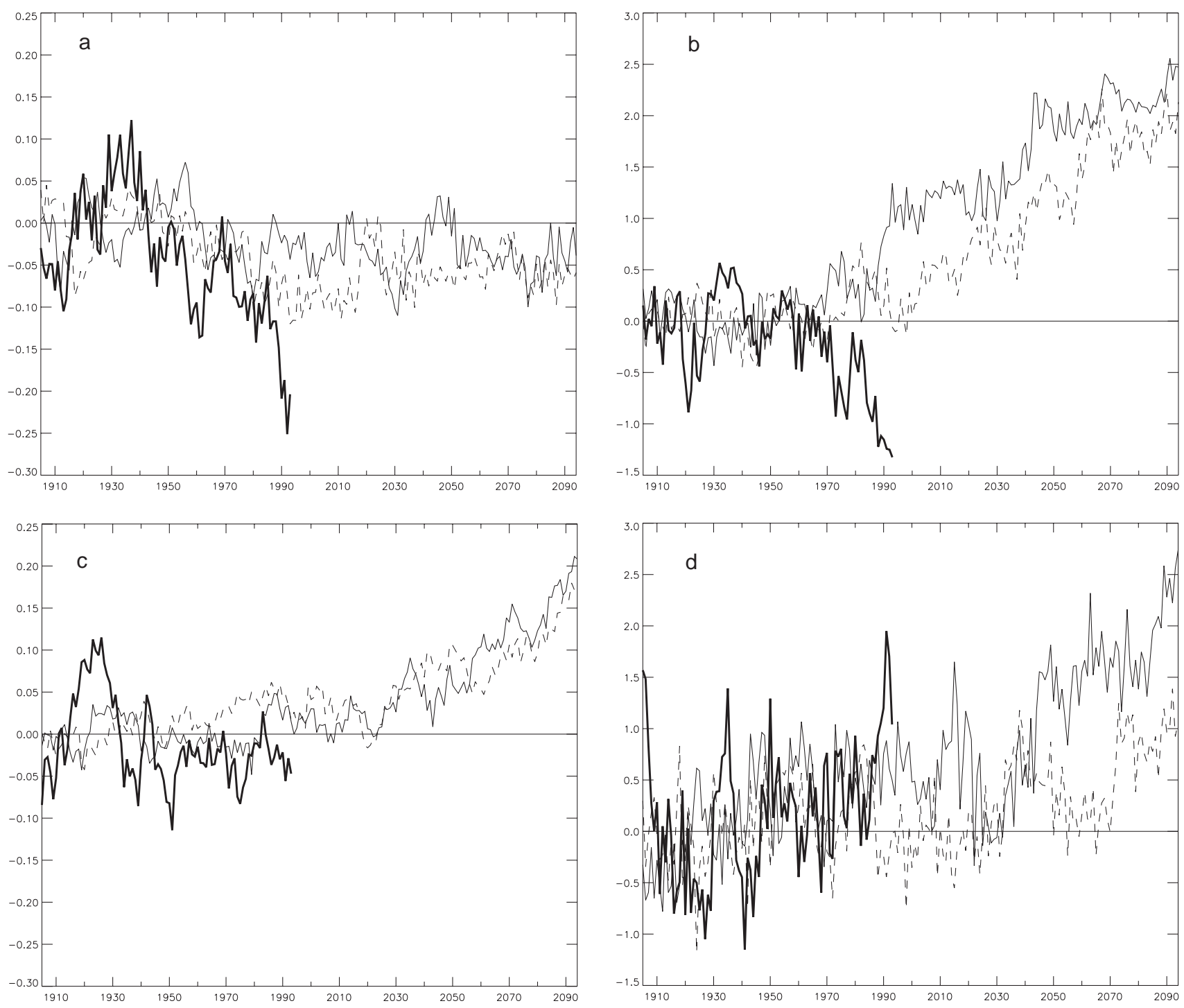

Fig. 6. Changes in frozen grid $(\mathrm{a}, \mathrm{c})$ amplitude $\left({ }^{\circ} \mathrm{C}\right)$ and $(\mathrm{b}, \mathrm{d})$ phase (days) of the annual cycle of $(\mathrm{a}, \mathrm{b}) \mathrm{NH}$ and $(\mathrm{c}, \mathrm{d}) \mathrm{SH}$ temperature: observed (thick lines) and means from the G (thin lines) and GS (dashed lines) ensembles

Phase delays are evident in the simulated annual cycle, particularly in sea-ice regions of the SH. Similarly, parts of the Arctic Ocean exhibit phase delays (especially for $\mathrm{G}$ results) where the phase is delayed by $\sim 6 \mathrm{~d}$. Within the tropics, blank simulated grid boxes are present where the phase of the cycle has switched to the alternative temperature peak of the semi-annual cycle. Still, it is possible to deduce a small number of regions where phase has advanced by 8 to $16 \mathrm{~d}$. Corresponding regions of phase advances are less evident in the observed results (indeed, the north Indian Ocean shows phase delays of a similar magnitude), and it is not possible to compare high-latitude changes, due to the lack of observational data. Advances of up to $4 \mathrm{~d}$ can be seen in western Russia, whilst regions of western Europe show slight phase delays. As with the amplitude, it is the observed change which exhibits the greatest magnitude of change, when a like-for-like comparison is made (the area-mean rms deviation for observations is 2.24, whilst for the $\mathrm{G}$ simulation it is 1.14 and for GS 0.96). The GS changes show a marginally stronger pattern correlation with observations than $\mathrm{G}$ changes, having a correlation coefficient of 0.16 , compared with 0.07 , although again, the significance of such relationships is difficult to estimate given the unknown number of degrees of freedom. 
a

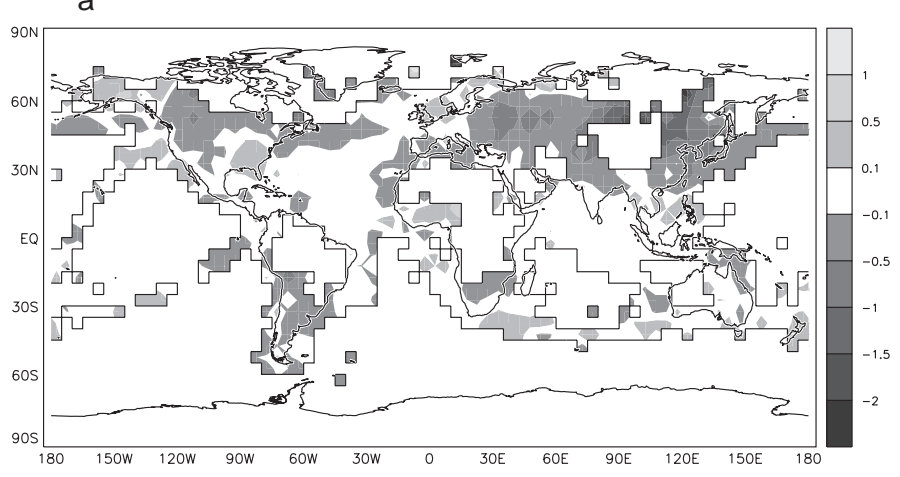

C

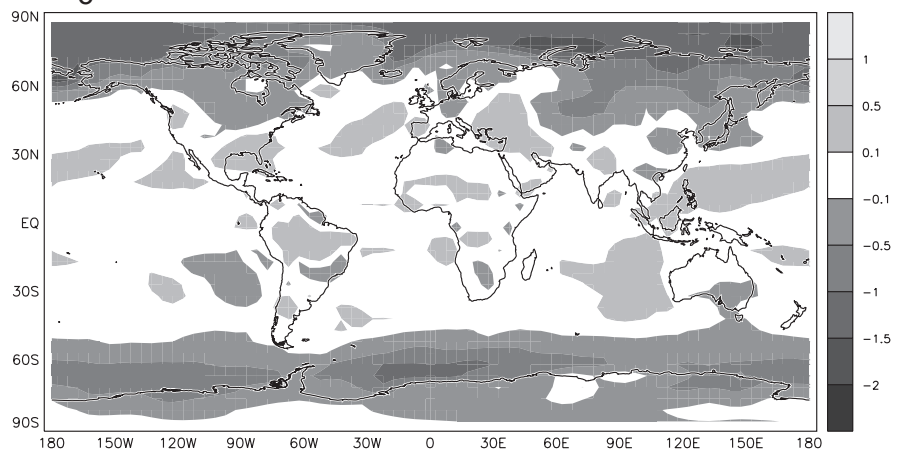

e

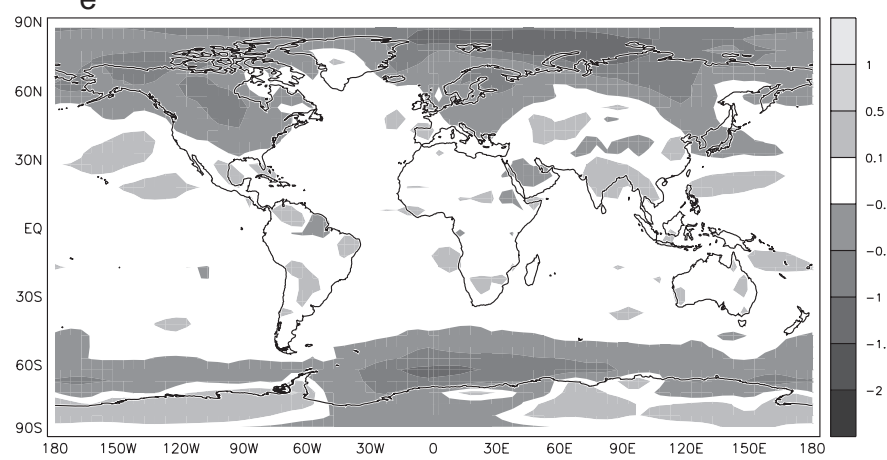

b

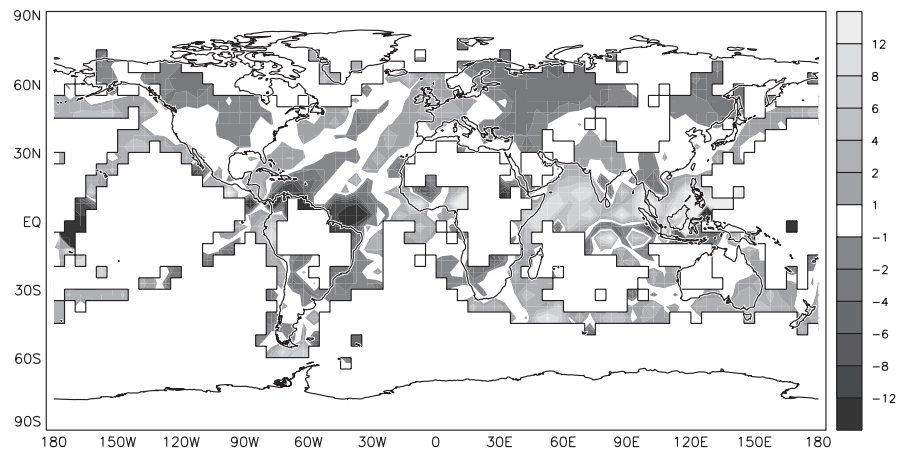

$\mathrm{d}$

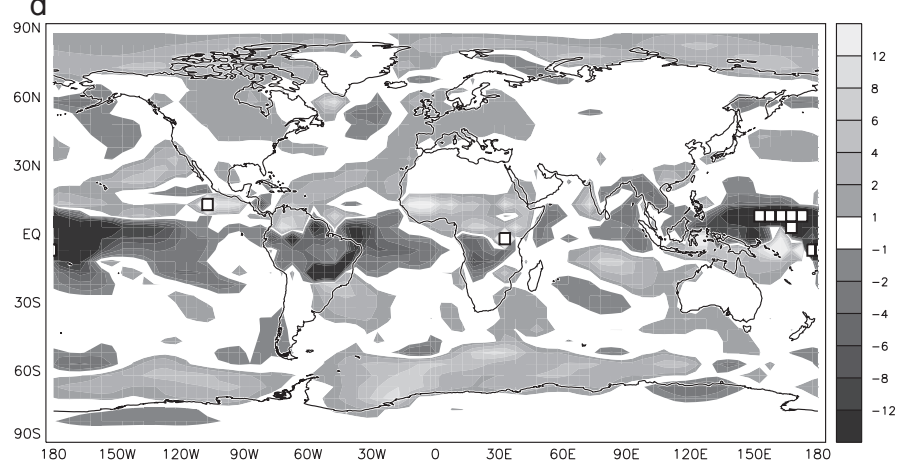

$\mathrm{f}$

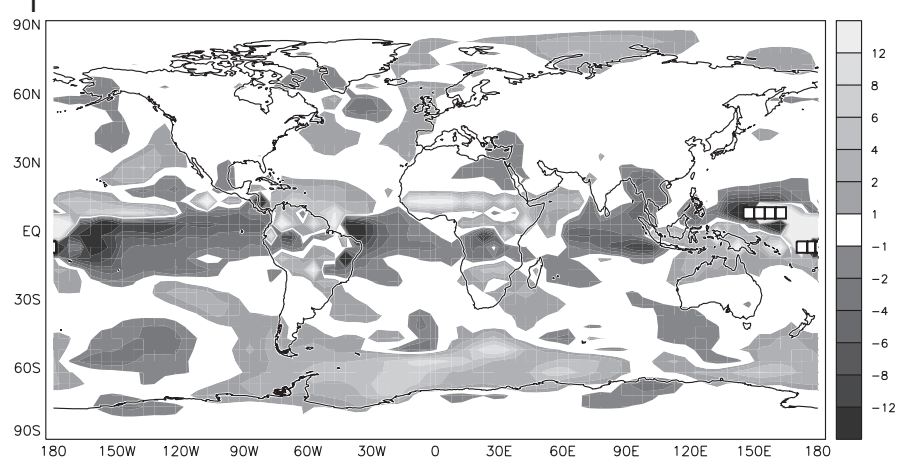

Fig. 7. (a,b) Observed, (c,d) G and (e,f) GS differences between 1909-1938 and 1969-1998 average annual cycle (a,c,e) amplitude $\left({ }^{\circ} \mathrm{C}\right)$ and $(\mathrm{b}, \mathrm{d}, \mathrm{f})$ phase (days)

\section{DISCUSSION AND CONCLUSIONS}

The phase and amplitude of the first harmonic of the annual temperature cycle were examined in observed and simulated data (from the HadCM2 climate model). There are inherent differences between simulated and observed annual cycles, especially in high-latitude regions. Some of these differences are related to a model underestimation of winter minimum temperatures in northern North America. Still, on a hemispheric scale, the simulated annual cycle seems a generally good representation of reality, in comparison with the observed 1961-1990 climatology, though a climate model without seasonally varying flux adjustments may be less successful.
Observed decreases during the 20th century in the $\mathrm{NH}$ annual cycle amplitude correspond well with simulated changes. The nature of these results compare well with the analysis of the GFDL model done by Mann \& Park (1996). However, the simulated decrease in the $\mathrm{SH}$ amplitude is not evident in the observed data set, though it remains within the range of natural variability. Application of a frozen grid mask precludes the possibility of changes in the spatial coverage of the observed data introducing artificial trends. The existence of a similar, yet larger, trend in the observed NH and amplitude, when using the frozen grid, suggests that the amplitude changes are bona fide. The size of these changes exceed simulated results for the same grid mask. The spatial pattern of observed changes 
shows that central and eastern Russia have seen the greatest decrease in amplitude. Meanwhile, simulated results suggest a more moderate response in these areas, with larger amplitude decreases in both northern and southern polar regions. Unfortunately, spatial coverage is insufficient at the start of the observed data set to validate model predictions for these regions.

Observed $\mathrm{NH}$ advances (obtained from either the full grid or the frozen grid) conflict with the simulated phase delays under greenhouse or greenhouse plus sulphate forcing, a feature consistent with results from the GFDL model (Mann \& Park 1996). The spatial pattern of simulated changes shows the Southern Ocean exhibiting the greatest delays, possibly related to the formation or decay of sea ice. A similar, but smaller, region of change also exists in the northern high latitudes, but it is not possible to compare these regional changes with the observed data set. The observed data set does, however, reveal a region of phase advance, located in western Russia, not captured in the simulations.

The sulphate effect (defined as the difference between the G and GS ensemble results) can be seen as a delay in the climate warming signal (and hence amplitude changes) superimposed upon a slightly larger decrease in amplitude in the $\mathrm{NH}$, where the direct sulphate-related reduction in net summer solar radiation occurs.

Two questions are raised in conclusion. First, it is important to establish why observed amplitude decreases (outside high latitude regions) are not present in simulated results. Do the observed trends represent a greenhouse gas signal expressed by mechanisms present in reality but absent from HadCM2, or are they the result of some alternative forcing (e.g. land cover changes, see Betts 2001) or internal variability such as the winter North Atlantic Oscillation? Investigation into observed trends of land surface variables (e.g. snow cover in high latitudes or soil moisture in drier regions) might help comprehension of the causal physics. Second, why are phase delays not apparent in observations? The absence of high latitude observational sampling may go some way to answering this question with respect to the results based on the full model output, but phase delays are similarly

Editorial responsibility: Hans von Storch, Geesthacht, Germany predicted when the simulations are masked by the frozen grid. Yet all that is observed is a phase advance (see also Mann \& Park 1996). Here too further investigation of surface variables might prove useful.

Acknowledgements. The Climate Impacts LINK Project (UK DETR contract number EPG 1/1/68) provided Hadley Centre model output. T.J.O. was supported by the UK Natural Environment Research Council (GR3/12107).

\section{LITERATURE CITED}

Betts RA (2001) Biogeophysical impacts of land use on present-day climate: near-surface temperature change and radiative forcing. Atmos Sci Lett 2:39-51

Johns TC, Carnell RE, Crossley JF, Gregory JM, Mitchell JFB, Senior CA, TettSFB, Wood RA (1997) The second Hadley Centre coupled ocean-atmosphere GCM: model description, spinup and validation. Clim Dyn 13:103-134

Jones PD, New M, Parker DE, Martin S, Rigor IG (1999) Surface air temperature and its changes over the past 150 years. Rev Geophys 37:173-199

Mann ME, Park J (1996) Greenhouse warming and changes in the seasonal cycle of temperature: model versus observations. Geophys Res Lett 23:1111-1114

May W, Shea DJ, Madden CGD (1992) The annual variation of surface temperatures over the world. NCAR Tech Note NCAR/TN-372+STR, National Center for Atmospheric Research, Boulder, $\mathrm{CO}$

Mitchell JFB, Johns TC, Eagles M, Ingram WJ, Davis RA (1999) Towards the construction of climate change scenarios. Clim Change 41:547-581

Pierce DW (1996) Reducing phase and amplitude errors in restoring boundary conditions. J Phys Oceanogr 26: $1552-1560$

Stott PA, Tett SFB, Jones GS, Allen MR, Ingram WJ, Mitchell JFB (2001) Attribution of twentieth century temperature change to natural and anthropogenic causes. Clim Dyn $17: 1-21$

Tett SFB (1997) Global and regional variability in a coupled AOGCM. Clim Dyn 13:303-323

Thompson R (1995) Complex demodulation and the estimation of the changing continentality of European climate. Int J Climatol 15:175-185

Thomson DJ (1995) The seasons, global temperature, and precession. Science 268:59-68

van den Broeke MR (1998) The semi-annual oscillation and Antarctic climate. Part 1: influence on near surface temperatures (1957-79). Antarct Sci 10:175-183

Wilks D (1995) Statistical methods in the atmospheric sciences. Academic Press, New York, p 325-341

Submitted: May 22, 2001; Accepted: November 18, 2001 Proofs received from author(s): June 21, 2002 